\title{
A Lightweight Approach to Performance Portability with targetDP
}

\author{
Alan Gray ${ }^{1}$ and Kevin Stratford ${ }^{1}$ \\ March 8, 2022
}

\begin{abstract}
Leading HPC systems achieve their status through use of highly parallel devices such as NVIDIA GPUs or Intel Xeon Phi many-core CPUs. The concept of performance portability across such architectures, as well as traditional CPUs, is vital for the application programmer. In this paper we describe targetDP, a lightweight abstraction layer which allows gridbased applications to target data parallel hardware in a platform agnostic manner. We demonstrate the effectiveness of our pragmatic approach by presenting performance results for a complex fluid application (with which the model was codesigned), plus a separate lattice QCD particle physics code. For each application, a single source code base is seen to achieve portable performance, as assessed within the context of the Roofline model. TargetDP can be combined with MPI to allow use on systems containing multiple nodes: we demonstrate this through provision of scaling results on traditional and GPU-accelerated large scale supercomputers.
\end{abstract}

\section{Keywords}

Performance Portability, Programming Models, HPC, GPU, Manycore, Computational Physics

\section{Introduction}

Each new generation of HPC system must offer an increase in the number of operations that can be performed per second. Performance advances in hardware are realized through parallelism, where the trend is not just to increase the number of compute nodes but also to increase the capability of each node through use of highly parallel devices such as Graphics Processing Units (GPUs) or manycore CPUs. The key challenge for application programmers is how to achieve "performance portability", such that a single, easily maintainable source code base can run with optimal performance across the range of modern parallel architectures.

Most HPC applications have, over decades of development targeting traditional CPU-based systems, grown to many thousands of lines of code written in base languages such as $\mathrm{C}, \mathrm{C}++$ and Fortran. Compiler technology cannot automatically transform such raw code into executables that can always perform well across such architectures, so some form of modernization is required at the source code level. In order to achieve performance portability, it is inevitable that the concept of abstraction must play a role in one form or another, to allow generic syntax to be mapped to a range of different executables. There are two potential pitfalls with this approach, though. The first is that any codegeneration software required to perform the mapping must be maintained along with the application, so it is important that the software sustainability problem is not just shifted from the application into the generator. It is very difficult to avoid this problem for any sophisticated code-generation mechanism. If a large community can reach agreement, then the maintenance can be shared, but of course even when achievable this can take time. The second potential pitfall is that the modernization necessary can be relatively disruptive, which can discourage developers from committing to the new approach.

In this paper we address these problems in a pragmatic way, where our abstraction is intentionally kept as simple and lightweight as possible. We demonstrate the effectiveness of this approach through performance portability results for real complex applications. The targetDP model (Gray and Stratford 2014) provides an abstraction layer which allows applications to target Data Parallel hardware in a platform agnostic manner, by abstracting the memory spaces and hierarchy of hardware parallelism. Applications written using targetDP syntax are performance portable: the same source code can be compiled for different targets (where we currently support NVIDIA GPU accelerators and modern multicore and manycore CPUs including the Intel Xeon Phi which rely on vectorization). TargetDP is implemented using a minimal set of C-preprocessor macros and library functions, so is easily maintainable, sustainable and extendable. There are no special datatypes, so it is possible to adapt incrementally and also inter-operate with other programming paradigms. The model is appropriate for abstracting the parallelism contained within each compute node, and can be combined with, e.g. MPI to allow use on systems containing multiple nodes.

The model and implementations were developed in codesign with a particular HPC application, Ludwig (Desplat et al. 2001), which uses lattice Boltzmann and finite difference techniques to simulate a wide range of complex

\footnotetext{
${ }^{1}$ EPCC, The University of Edinburgh

Corresponding author:

Alan Gray, EPCC, The University of Edinburgh

Edinburgh, EH9 3FD, UK

Email: a.gray@ed.ac.uk
} 
fluids. However, we demonstrate general applicability using the separate MILC code (The MILC Collaboration 2014), which simulates fundamental particle physics phenomena through Lattice QCD techniques. TargetDP is particularly suited to the structured grid techniques employed by both of these applications since the regularity and parallelism can be exploited in a straightforward manner. Therefore, whilst at present the model can be thought of as being specific to this computational domain, it should also applicable to a wider class of data parallel problems.

In Section 2 we describe these applications, focusing on the test cases used for performance results, and we also discuss other approaches to performance portability. In Section 3, we present the abstractions used to achieve performance portability. We go on to present singleprocessor results in Section 4, where we analyse performance across architectures following the Roofline model (Williams et al. 2009) (which gives standard guidelines for comparing against the capability of the hardware). We extend the analysis to include scaling over multiple nodes of supercomputers in Section 5, through the combination of targetDP and MPI.

\section{Background}

\subsection{The Applications}

Many scientific and engineering problems are made tractable through the discretization of space and time, to allow representation and evolution within a computer simulation. In this paper we focus on two such applications, Ludwig and MILC (both of which are open source, use C as a base language and MPI for node-level parallelism).

\subsubsection{Ludwig}

Many substances which are neither solids nor simple fluids can be classed as "soft matter", also known as "complex fluids". Familiar examples include foodstuffs, lubricants, cosmetic and health-care items, bodily fluids (such as those found in the joints) and, pertinent to this paper, liquid crystals (LCs). The versatile Ludwig simulation package (Desplat et al. 2001) is a vital link between theory and experiment in enabling new and improved soft matter materials. Like many such applications, Ludwig represents space as a $3 \mathrm{D}$ structured grid (or lattice), where the physical system, at a certain point in time, is represented by a set of double precision values at each lattice point. The lattice Boltzmann (LB) method (Succi 2001) is used to evolve the hydrodynamics in a standard timestepping manner. Ludwig, however, can simulate not just simple but complex fluids; the hydrodynamical evolution is coupled with other finite difference techniques to properly represent the substance under investigation.

LCs are well known for their utility in displays, but are also prevalent in a variety of other technological items and natural systems. There is still much to be understood about the range of possible LC systems, which are a key focus of interest in current research using coarse-grained methods (Henrich et al. 2013)(Tiribocchi et al. 2014). The LC simulation, which comprises one of the testcases used later in this paper, couples an "order parameter" field (a $3 \times 3$ tensor, which is symmetric and traceless) representing the composition and structure of the crystal, and a "distribution" field representing the flow of the fluid. The former is evolved via an advection-diffusion equation appropriate for rod-like molecules, and the latter via $L B$. They interact through a local force, derived from the former, which acts on the latter at every timestep of the simulation. The main computational components of each timestep are as follows, where the names given in quotes are those used later in this paper when presenting performance results. The "Collision" models the interaction of the individual fluid molecules and the "Propagation", which mimics the convection of the fluid, involves displacing the fluid data one lattice spacing in the appropriate direction. The force which couples the order parameter with the distribution is calculated as the divergence of the "Chemical stress", which in turn is calculated as a function of the order parameter field and its "Order Parameter Gradients" derivatives. The "LC Update" involves evolution of the order parameter itself, using a finite difference implementation of the Beris-Edwards model with the Landau-de Gennes free energy functional (Beris and Edwards 1994)(de Gennes and Prost 1995). The "Advection" involves calculating the flux in the order parameter due to the advective bulk flow, and "Advection Boundaries" includes the effects of any boundary conditions to this.

To allow utilization of multi-node computing architectures, Ludwig is parallelized using domain decomposition and MPI in a standard way. "Propagation", "Advection", "Advection Boundaries", and "Order Parameter Gradients" involve updates based on neighbouring lattice site data, so are classed as stencil operations, whereas "Collision", "Chemical Stress" and "LC Update" involve operations local to each lattice site. To allow for the former, each local sub-domain is surrounded by a halo region populated using neighboring sub-domain data through MPI communications. In recent years we have adapted Ludwig for use on multiple GPUs in parallel (as described in (Gray et al. 2012), (Gray et al. 2015) and (Gray and Stratford 2013)), which has enabled an improved understanding of the interactions between LCs and large colloidal particles (Stratford et al. 2015).

\subsubsection{MILC}

Matter consists of atoms, which in turn consist of nuclei and electrons. The nuclei consist of neutrons and protons, which comprise quarks bound together by gluons. The theory of how quarks and gluons interact to form nucleons and other elementary particles is called Quantum Chromo Dynamics (QCD). For most problems of interest, it is not possible to solve QCD analytically, and instead numerical simulations must be performed. Such Lattice QCD calculations are known to give excellent agreement with experiment (Davies et al. 2004), at the expense of being very computationally intensive.

The MIMD Lattice Computation (MILC) code is a freely available suite for performing Lattice QCD simulations, developed over many years by a collaboration of researchers based in the US (The MILC Collaboration 2014). The test case used in this paper is derived from the MILC code (v6), and forms a component Unified European Application Benchmark Suite (UEABS), a set of application codes designed to be representative of HPC usage in the European 
Union (Bull 2013). This consists of the inversion of the Wilson Dirac operator using the conjugate gradient method. The work presented in this paper is part of a project to extend the UEABS suite to accelerators.

Spacetime is discretized into a 4D grid, and at each point on the grid exists a set of values that represent the quark and gluon content at that point. These structures are relatively small (compared to the size of the grid) complex vectors and matrices, and the conjugate gradient algorithm involves correspondingly small linear algebraic operations at each point on the grid. With reference to the labels used for performance analysis later in this paper, "Extract" involves extracting the quark field from one representation to another, and "Extract and Mult." involves a similar quark field manipulation together with a matrix-vector multiplication to interact with the gluon field. The "Insert and Mult" and "Insert" parts involve the reverse process. "Scalar Mult. Add" involves a scalar multiplication and vector addition for the quark field. These operations are all local to each grid point, but the "Shift" operation involves shifting the quark field data in each direction, which is a stencil operation. When running on a single processor, this just involves local memory copy operations but, when operating with nodelevel parallelism, MPI is used within this operation to buffer and move the data that must cross subdomain boundaries.

\subsection{Other Approaches to Performance Portability}

The use of a low-level native programming model, e.g. CUDA for NVIDIA GPUs, permits excellent performance on the native architecture but lacks portability.

The OpenCL standard by the Khronos group (Khronos OpenCL Working Group 2015) gives a similar model to CUDA but with improved portability, particularly to AMD GPUs, and in principle to X86 CPUs including Xeon Phi. Intel do not currently plan to support OpenCL, however, on the next generation of Xeon Phi (Knights Landing). It is also relatively low level and complex, and can have performance overheads relative to native approaches (although the situation is improving (McIntosh-Smith et al. 2014)). The SYCL standard (Khronos OpenCL Working Group - SYCL Subgroup 2015), also by Khronos, has recently emerged: this aims to be higher level and more productive, particularly for $\mathrm{C}++$ applications. There also is active research into automatic generation of hardwarespecific OpenCL code, e.g. with functional programming techniques and rewrite rules (Steuwer et al. 2015). The use of OpenCL, within the types of applications discussed in this paper, would mean increased complexity and compromised portability and performance, compared with targetDP.

The OpenACC directive-based standard (The OpenACC Standard Committee 2015) is designed to provide a higherlevel and more user-friendly alternative to CUDA/OpenCL for development of GPU-accelerated applications. The idea is that a greater onus is put on the compiler to automatically manage data movement and computational offloading, with help from user-provided directives. The model is prescriptive, such that the compiler is free to map parallelism and data movement to the specific hardware in an automatic manner (although a range of directives and clauses are available providing the user with the ability to override.) OpenACC offers portability across NVIDIA and AMD GPUs, and has recently expanded support to multicore CPUs. However, there is still no support for Intel Xeon Phi, and Intel do not participate on the OpenACC committee. Whilst OpenACC is not yet a viable option for true performance portability, it will be interesting to monitor progress. A key concern is whether the level of flexibility available is adequate for the intricacies associated with real complex applications (e.g. the interaction of particles with liquid crystals in Ludwig which requires very fine-grained control over movement of data between memory spaces to ensure good performance). Similarly to OpenACC, The OpenMP directives-based standard (OpenMP Architecture Review Board 2015) has, since version 4.0, included support for accelerators. OpenMP aims to be more portable than OpenACC, including participation from Intel and support for the Xeon Phi. However, the required directives actually differ across architectures, so the approach ultimately lacks portability. Similarly to OpenACC, the relatively high-level OpenMP model offers productivity at the expense of user control (and hence often performance).

Alternatively, a separate source-to-source generator (e.g. a preprocessor, compiler or library) can be used to transform generic appropriately defined syntax into multiple forms, which can each be compiled by the standard compiler for the specific target architecture. Kokkos (Edwards et al. 2012) and RAJA (Hornung et al. 2014), both C++ abstraction frameworks, have conceptual similarities to the work presented in this paper, since they provide suitable abstractions for node-level parallelism and data movement to allow portable performance across modern architectures. These are much more sophisticated than targetDP since they aim to be general purpose models and support a wide range of application areas. The caveat, however, is the disruption required to adapt legacy applications (particularly those which do not already use $\mathrm{C}++$ ). Additionally, users are often hesitant to commit to third-party models and libraries which do not form any standard so have no guaranteed longevity. The developers are addressing the latter by lobbying for much of the models to be integrated into the $\mathrm{C}++$ standard. The work described in this paper, conversely, exploits the domain specificity (to grid-based codes) to retain simplicity, so these issues are much less severe. Other frameworks such as OCCA (Medina et al. 2014) and HEMI (Harris 2016) are alternative $\mathrm{C}++$ based models, which have varying levels of support for explicitly targeting SIMD units.

Legion (Bauer et al. 2012) is another very sophisticated model. This not only provides portable abstractions, but also allows the programmer to express tasks and dependencies which, through a runtime system, can be automatically executed with optimal ordering, dependent on the hardware resource in use. This is very powerful in extracting performance, but even more disruptive to the programmer that the approaches discussed above. Legion is really designed as a low level interface for other domain specific languages to be built on. Recently, however, the Regent compiler (Slaughter et al. 2015) has been introduced which generates Legion code from a higher-level model.

So clearly, the best option for a programmer wishing to invest in code modernization depends on the application. 
Given our pragmatic and simple approach, one aim of this paper is to clearly describe, in an accessible manner, those concepts which are common, such as the abstraction of memory accesses and data parallel operations, the expression of data locality, and the resulting performance portability achieved. We hope this is of interest even to those readers who decide to follow one of the other available development routes.

\section{3 targetDP}

In this section we describe the targetDP programming model (first introduced in (Gray and Stratford 2014)). The aim is to provide, in the simplest possible way, an abstraction that can allow the same data-parallel source code to achieve optimal performance on both CPU-like architectures (including Xeon Phi) and GPUs. Before describing our model, we first give details on how we abstract memory accesses through a simple layer. This is necessary to allow architecturespecific data-layouts, which are vital for memory bandwidth performance.

\subsection{Data Layout}

To facilitate optimal memory bandwidth, it is crucial that the layout of data in memory is tailored for the specific memory access patterns that result from the execution of a targetDP application on any specific architecture.

For many grid-based applications, the simulation data is "multi-valued": comprised from multiple numerical values located at each point on the grid, and how we choose to store this in memory can have a dramatic effect on performance. To illustrate the available options, a useful analogy is that of red (r), green (g) and blue (b) values collectively representing each pixel of an image. For brevity, let's assume that our image is very small at only 4 pixels. The naive way to store the entire image is $|\mathrm{rgb}| \mathrm{rgb}|\mathrm{rgb}| \mathrm{rgb} \mid$. This is known as the Array of Structures (AoS) format since we are storing the rgb values consecutively as each entry of an array of pixels. An alternative is the Structure of Arrays (SoA) storage scheme $\mid$ rrrr|gggg|bbbb|. These are the two opposite extremes of a more general scheme: the Array of Structures of (short) Arrays (AoSoA) format, where the length of the short array can vary. For example, $\| \mathrm{rr}|\operatorname{gg}| \mathrm{bb}|||\mathrm{rr}| \mathrm{gg}|\mathrm{bb}| \mid$, which has a short array length of 2 , is a layout in which we store the first 2 values of each of red, green and blue, then repeat for the next 2 of each. The AoS and SoA formats described above have a short array length of 1 and 4 respectively in this case.

To represent such multi-dimensional structures in $\mathrm{C}$ or $\mathrm{C}++$, it is usually most straightforward to use 1-dimensional arrays in the application and to "flatten" accesses through linearization of the indices. Let ipix and irgb denote the pixel and rgb value indices of this 2-D problem respectively, and $\mathrm{N}$ be the total number of pixels (with, of course, 3 being the total number of rgb components). Then, if using the AoS scheme we access the image with code such as

image [ipix*N+irgb]. The alternative SoA scheme would instead use image [irgb*3+ipix], and AoSoA would use (where the short array length is specified through the SAL preprocessor-defined integer constant).

For most lattice-based scientific simulations, including those discussed in this paper, the main bottleneck is memory bandwidth. The specific layout of data in memory has a major effect on memory bandwidth performance and unfortunately the best layout differs across architectures. Therefore, for performance portability it is required memory accesses should not be explicitly written, as above, within the application. Instead they should be abstracted through an intermediate layer, in which the optimal layout can be specified through a configuration option without any changes to the application itself.

Following the philosophy of this paper, we achieve this in a simple way. We define macros using the Cpreprocessor to provide the required memory addressing layer. At the application level, our accesses become, e.g., image [INDEX (irgb, ipix)], where different versions of the INDEX macro can expand to match different linearizations (or, equivalently, the single generic AoSoA version can be used with different short array lengths).

We can follow the practice described in this section for the multi-dimensional data structures that naturally exist in real structured grid problems, which have one dimension corresponding to the grid points (c.f. the pixel dimension) and one or more dimensions correspond to the components stored at each grid point (c.f. the rgb values). Note that in practice we also pass extent values into the macros to allow flexibility regarding grid dimensions.

\subsection{Data Parallel Abstractions}

The execution time of grid-based applications is typically dominated by the parts of the code that perform dataparallel operations across the grid: at each point on the grid, the multi-valued data resident at that point is updated (sometimes using data from neighbouring grid points). We use the the terminology "host" to refer to the CPU on which the application is running, and "target" refers to the device targeted for execution of these expensive operations. When the target is a GPU, there is clearly a distinction in hardware between the host and target. But the host and target can also refer to the same hardware device, for instance when we are using CPU (or Xeon Phi) architectures. Importantly, we always retain the distinction of host and target in the application, to allow a portable abstraction.

The aim of targetDP is to provide a lightweight abstraction that can map data-parallel codes to either CUDA or OpenMP, whilst allowing good vectorization on CPU-like architectures. Note that, since the abstraction layer maps directly to the underlying model in each case, by design there is negligible overhead compared to an equivalent native implementation.

\subsubsection{Thread-level Parallelism}

Consider a serial $\mathrm{C}$ code which involves a data-parallel loop over $\mathrm{N}$ grid points, e.g.

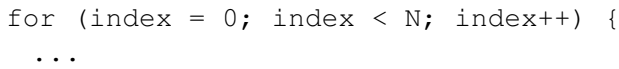

The ... refers to whatever operation is performed at each grid point. An OpenMP implementation of this code would 
instruct the compiler to decompose the loop across threads with use of a pragma, e.g.

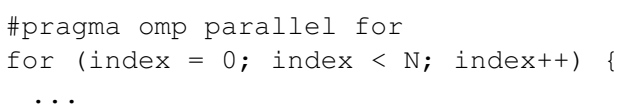

The thread-level parallelism in CUDA does not involve the concept of loops. Instead, the grid-based operation becomes a kernel to be executed in parallel by a number of threads, e.g.

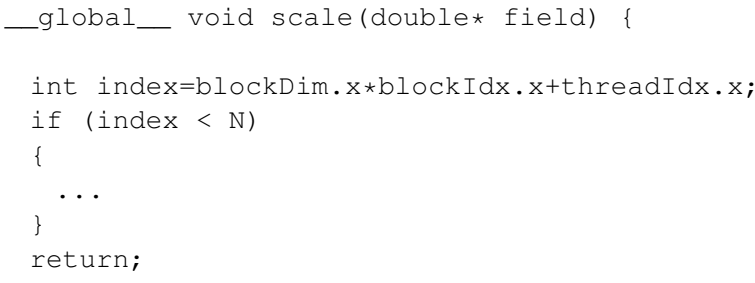

The operation must be contained within a function, where the global_ keyword specifies it should be compiled for the GPU, and CUDA internal variables are used to retrieve a unique index for each thread.

So, therefore, we can provide an abstraction that can map to either OpenMP or CUDA by introducing the targetDP syntax:

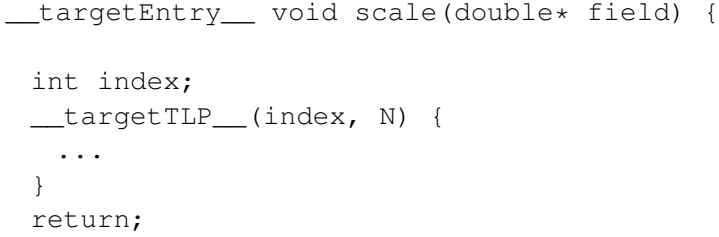

targetentry_ and _ _targetTLP_ are Cpreprocessor macros. The former is used to specify that this function is to be executed on the target and it will be called directly from host code (where the analogous _target__ syntax is for functions called from the target). For implementation in CUDA it is mapped to global_, so the function becomes a CUDA kernel, and for the $\mathrm{C}$ implementation the macro holds no value so the code reduces to a regular function. We expose the grid-level parallelism as thread-level parallelism (TLP) through the _target TLP_(index, N) syntax, which is mapped to a CUDA thread look-up (CUDA implementation), or an OpenMP parallel loop (C implementation). In the former case, the targetDP example therefore maps exactly to the CUDA example. For the latter, the only difference to the OpenMP example is that the code is now contained in a function.

\subsubsection{Instruction-level Parallelism}

We must also be able to achieve good vectorization on CPUlike architectures. The compiler will try to create vector instructions from innermost loops, but the problem with the above is that the extent of the innermost loop is dependent on the application, so does not necessarily map well to any specific hardware vector length (and furthermore not all such loops over multi-valued data are parallel in nature). To give a simple example, imagine that . . . is given by

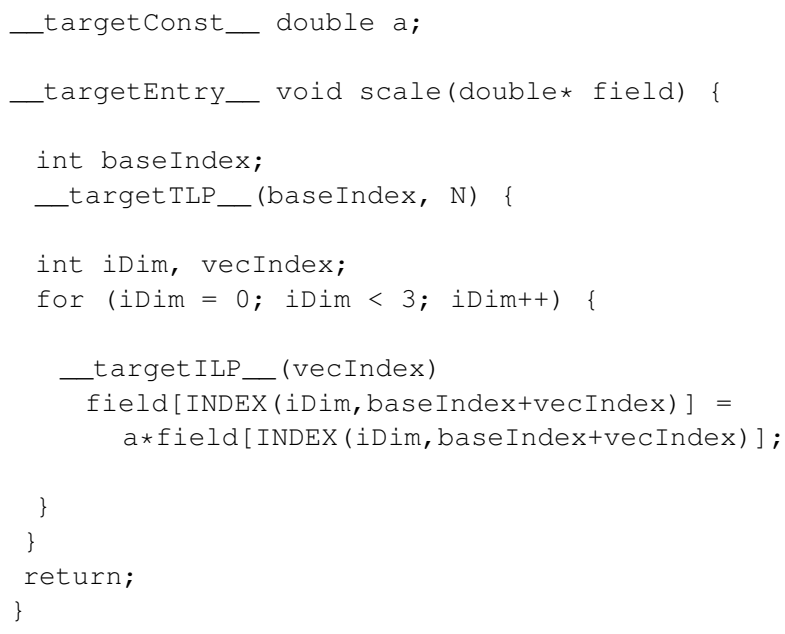

Figure 1. A simple example of a targetDP kernel.

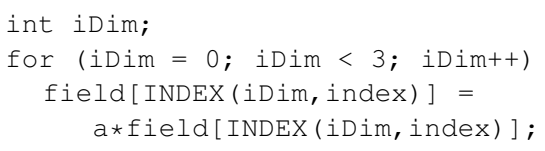

The field data structure has 3 components at each grid point, and an extent of 3 for the innermost loop is not optimal for creating vector instructions of size, e.g. 4 or 8 for CPU or Xeon Phi (which, when using double precision is the natural vector length for these architectures which feature 256-bit AVX and 512-bit IMCI instructions), respectively. The solution is that we can allow striding within our implementation of __target TLP_, such that each thread works, not on a single lattice site, but a chunk of lattice sites. Then, at the innermost level, we can re-introduce this grid-level parallelism for the compiler to vectorize. The size of the chunk (i.e. length of the stride), which we call the "Virtual Vector Length" (VVL), can be tuned to the hardware through a targetDP configuration option at compile time. The new instruction-level parallelism (ILP) is specified using the _targetILP_ syntax. When $V V L>1$, then targetILP_ is mapped to a short loop over the sites in the chunk, augmented with the OpenMP SIMD directive. Since the loop extent, VVL, appears as a constant to the compiler (and is chosen to be a suitable value), vectorization is straightforward. The ILP syntax, therefore, has an associated index that ranges between 0 and VVL-1, such that it can be used as an offset when combined with the TLP thread index in accessing arrays. The final targetDP implementation for this example is given in Figure 1. Note that we also include the definition of the constant that is used in the multiplication: the _targetConst_ macro maps to _constant__ in the CUDA implementation (and holds no value for the $\mathrm{C}$ implementation) to allow use of the constant memory cache on the GPU.

\subsubsection{Memory Management}

The scale function is called from host code as in Figure 2. This illustrates how the model draws a distinction between the memory space accessed by the host and that accessed by the target. The targetMalloc and targetFree API functions wrap cudaMalloc and cudaFree for the CUDA implementation, and regular malloc and free 


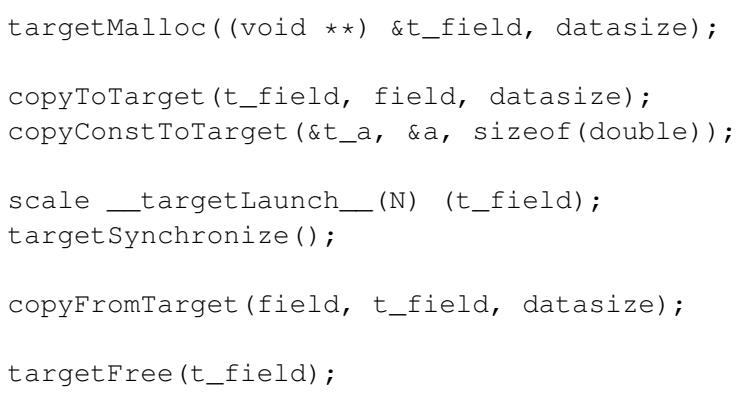

Figure 2. The host code used to call the targetDP kernel.

for the $\mathrm{C}$ implementation *. Similarly, the copy * routines abstract data transfer in a portable fashion. It is assumed that code executed on the host always accesses the host memory space, and code executed on the target (i.e. within target functions) always accesses the target memory space. The host memory space can be initialized using regular $\mathrm{C} / \mathrm{C}++$ functionality (not shown here). For each dataparallel data structure, the programmer should create both host and target versions, and should update these from each other as and when required. Data parallel operations are achieved through passing target pointers into target functions. The _targetLaunch__ macro maps to the regular CUDA launch syntax in the CUDA implementation, and holds no value in the $\mathrm{C}$ implementation, and similarly for targetsyncronize.

For the $\mathrm{C}$ implementation, the host and target versions of data structures will exist in the same physical memory space. The host implementation can implement these as physically distinct, or can instead use pointer aliasing: the model is agnostic to this. Note that this explicit memory model will map well to future CPU-like architectures, including the next generation of Xeon Phi, "Knights Landing", which features a high-bandwidth stacked memory space in addition to regular DRAM.

In applications, it is often necessary to perform reductions, where multiple data values are combined in a certain way. For example, values existing on each grid point may be summed into a single total value. The targetDP model supports such operations in a simple but effective way. It is the responsibility of the application to create the array of values (using standard targetDP functionality) to act as the input to the reduction operation. The application can then pass this array to the API function corresponding to the desired reduction operation (e.g. targetDoublesum for the summation of an array of double-precision values). If the required reduction operation does not yet exist, the user can simply extend the targetDP API using existing functionality as a template.

In this section we have illustrated the basic key functionality. Obviously, real applications are much more complex that the example given above, and correspondingly there exists a range of additional targetDP functionality, as detailed in the Specification (Gray and Stratford 2016). In particular, we provide functions that allow optimal performance when data transfers between host and target can be restricted to specific subsets of grid points.

\begin{tabular}{|c|c|c|c|}
\hline Processor & Product Details & $\begin{array}{c}\text { Peak Perf. } \\
\text { (Dbl. Prec.) }\end{array}$ & $\begin{array}{c}\text { Stream Triad } \\
\text { (Measured) }\end{array}$ \\
\hline \hline Ivy-Bridge & $\begin{array}{c}\text { Intel Xeon E5-2697 v2 } \\
\text { 12-core CPU @ 2.70GHz }\end{array}$ & 259 Gflops & $49.8 \mathrm{~GB} / \mathrm{s}$ \\
\hline Haswell & $\begin{array}{c}\text { Intel Xeon E5-2630 v3 } \\
\text { 8-core CPU @ 2.40GHz }\end{array}$ & 154 Gflops & $40.9 \mathrm{~GB} / \mathrm{s}$ \\
\hline Interlagos & $\begin{array}{c}\text { AMD Opteron 6274 } \\
\text { 16-core CPU @ 2.20GHz }\end{array}$ & 141 Gflops & $32.4 \mathrm{~GB} / \mathrm{s}$ \\
\hline Xeon Phi & $\begin{array}{c}\text { Intel Xeon Phi 5110P } \\
\text { 60-core CPU @ 1.053GHz }\end{array}$ & 1.01 Tflops & $158.4 \mathrm{~GB} / \mathrm{s}$ \\
\hline K20X & Nvidia Tesla K20X GPU & 1.31 Tflops & $181.3 \mathrm{~GB} / \mathrm{s}$ \\
\hline K40 & Nvidia Tesla K40 GPU & 1.43 Tflops & $192.1 \mathrm{~GB} / \mathrm{s}$ \\
\hline
\end{tabular}

Table 1. Technical details of the processors used in the performance analysis.

\section{Performance}

In this section, our main aim is to demonstrate that use of targetDP allows, for each application, a single version of source code to be performance portable across the range of relevant modern hardware architectures. We first present the performance, for each of the Ludwig and MILC codes, across different processors. We then go on to compare these observations to hardware capability, using the Roofline model to guide us.

Details of the processors used are given in Table 1. We include regular multi-core CPUs, the Intel Xeon Phi manycore CPU and NVIDIA GPUs. This choice naturally includes those processors present in the supercomputers on which we will extend our analysis in Section 5: Ivy-bridge, Interlagos and K20X. Haswell and K40 are newer lines than Ivy-Bridge and K20X respectively, so are included give assurance that our results are representative of modern technology.

To permit a clear comparison of the capabilities of the different processor architectures we restrict our analysis, in this section, to that using a single processor, be that a CPU or GPU. This means that, for example, we will compare a single Ivy bridge CPU with a single K40, where each is fully utilized. In section 5, where extend our analysis to multinode systems, we will compare on a node-by-node basis, with each node fully utilized, to give a comparison which is more natural considering normal utilization of such systems.

For the CPU and Xeon Phi (where the latter is in the "native" mode of use so can be treated on the same footing as a regular CPU) we use the $\mathrm{C}$ implementation of targetDP, which maps to OpenMP (with the number of threads set to best utilize the number of cores, or virtual hyperthreaded cores if the latter is beneficial) in each case. Note that the 16-core Interlagos processor is actually comprised from two 8-core chips in the same socket, so has two NUMA regions. Having tested the different options of mapping to this architecture, we use the best performing configuration of two MPI tasks each controlling 8 OpenMP threads (restricted to their respective NUMA regions). We use the CUDA implementation of targetDP for GPU results, with the number of CUDA threads per block set at 128 (which,

* Note that, as described in the Specification (Gray and Stratford 2016), we also support targetCalloc which is analogous but for allocation with initialization to zero, and targetMallocUnified/targetCallocUnified which can take advantage of modern unified memory (although we do not use the latter in the benchmarks presented in this paper). 

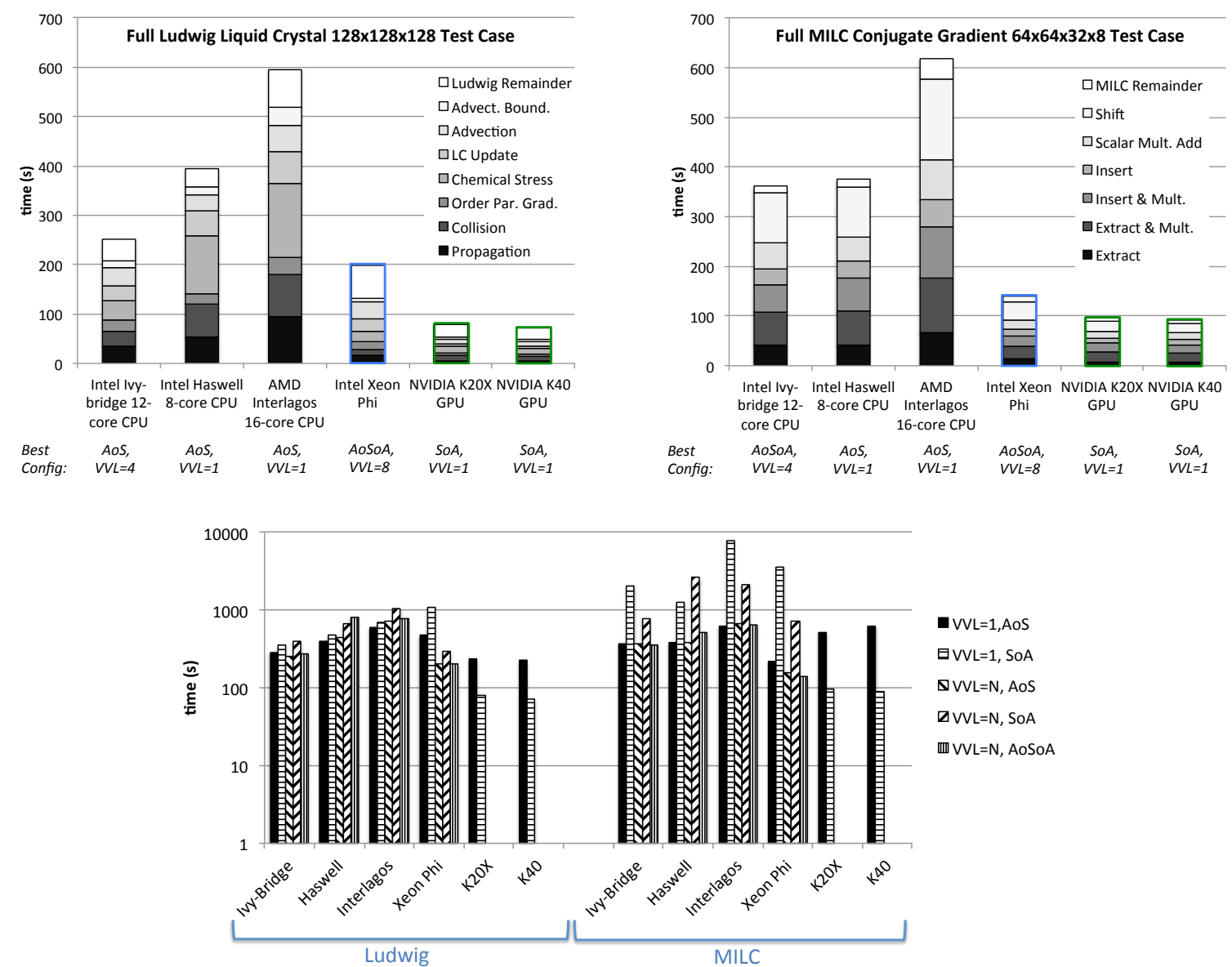

Figure 3. The time taken by the full Ludwig Liquid Crystal (top left) and MILC (top right) test cases on CPU, GPU and Xeon Phi architectures, where timings are decomposed into the different parts of the code. The same targetDP source code is used in each case and the best data layout and explicit vectorization configuration settings are shown, where variation across these options is shown in the bottom graph.

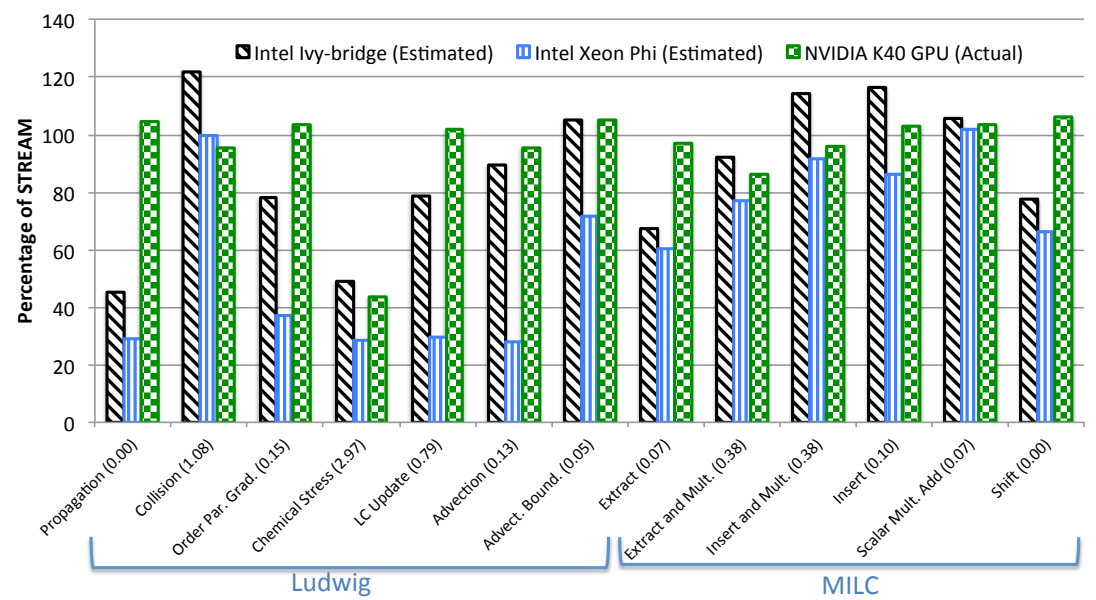

Figure 4. The memory bandwidth obtained by each Ludwig and MILC kernel, presented as a percentage of that obtained by the STREAM triad benchmark, across CPU, Xeon Phi and GPU architectures. For the application kernels, the GPU results are obtained using the NVIDIA profiler, and the other results are estimated from these using ratios of kernel time results to scale. The operational intensity of each kernel is given in brackets after the kernel name.

through trial and error, has been found to be a wellperforming value).

In Figure 3 we show the time taken for the full Ludwig (top left) and MILC (top right) application test cases, across the different architectures, where the testcase size (for each application) has been chosen to fit onto a single processor. These results include all overheads and have been run for 1000 timesteps (Ludwig) or conjugate gradient iterations
(MILC): this arbitrary number is chosen to be large enough to be representative of production usage (and smooth out any fluctuations), where each test is performed on an otherwise unoccupied node. The results in the figure are decomposed into the main kernels, plus the remainder which includes all other sections of the code (including PCI-express data transfer overheads in the GPU case). 
Considering the traditional CPU results it can be seen that, overall, the Ivy-Bridge has the performance advantage over the Haswell, where the difference is more pronounced for Ludwig. The AMD processor is seen to perform significantly less well than both its Intel counterparts. Now, when we extend our analysis to the remaining processor types, we clearly see the advantages offered by these modern devices. The GPUs are between 3 and 4 times as fast as the Ivy-Bridge, for both Ludwig and MILC. The Xeon Phi performance is seen to be significantly better than Ivy-bridge but worse than the GPU; better for MILC than for Ludwig.

As discussed in Section 3, the optimal choice of data layout and Virtual Vector Length (VVL) varies across architectures, and targetDP allows these options to be tuned. The above results are for the best performing choices, details of which are included underneath each column. The bottom graph in Figure 3 shows the how the performance varies with such choices (noting the log scale). When we set VVL to some value greater than one, we are introducing explicit vectorization by presenting grid-level parallelism at the innermost level. Conversely, when we set $\mathrm{VVL}=1$, then we revert to the naïve case where we have no explicit vectorization, and the compiler instead generates vector instructions, where possible, from the operations conducted on each grid point. For the CPU and Xeon Phi architectures, results are included for $\mathrm{VVL}=\mathrm{N}$, where, in double precision, $\mathrm{N}$ is naturally 4 for CPU and 8 for Xeon Phi given the instructions supported by these architectures (256-bit AVX and 512-bit IMCI respectively). It can be seen for the CPU results that there is no dramatic advantage from such explicit vectorisation: the compiler can typically do a reasonable job of finding vectorization implicitly. For the Xeon Phi, however, it is very clear that explicit vectorization is required to get optimal performance, otherwise the degradation is several-fold. Note that 512-bit instructions will soon propagate into future "traditional" CPU technologies, so these results indicate that such explicit vectorization will soon be vital across all modern CPUs. The use of a data layout which does not match the architecture-specific access pattern can be very detrimental. For example, for the MILC Xeon Phi $V V L=8$ case, use of the SoA layout results in a dramatic 5-fold slowdown overall relative to the natural AoSoA layout (and the slowdown for AoS is more modest but still significant at $12 \%$ ). GPUs clearly perform best with SoA data layout (which permits memory coalescing); the wrong layout can have dramatic effects, e.g. the MILC case is 7-fold slower with AoS. It is known that in some cases, it can be beneficial to include explicit vectorization in GPU kernels (Volkov 2010), but we do not find any advantage in doing so for the test cases presented in this paper. Overall, it is clear that the specific configuration is important for performance, and the flexibility offered by our model allows the user to experiment to find the best options on any specific architecture.

A standard methodology for comparing observed performance to the capability of the hardware involves the "Roofline" model as given in (Williams et al. 2009). This uses the concept of "Operational Intensity" (OI): the ratio of operations (in this case double precision floating point operations) to bytes accessed from main memory. The OI, in Flops/Byte, can be calculated for each computational kernel. A similar measure (also given in Flops/Byte), exists for each processor: the ratio of peak operations per second to the memory bandwidth of the processor. This quantity, which gives a measure of the balance of the processor, is known as the "ridge point" in the Roofline model. Any kernel which has an OI lower than the ridge point is limited by the memory bandwidth of the processor, and any which has an OI higher than the ridge point is limited by the processor's floating point capability. Table 1 gives peak performance values for each processor, together with memory bandwidth measurements obtained through the standard STREAM triad benchmark (McCalpin 1995). Dividing the former by the latter, the ridge points for the Ivy-bridge, Xeon Phi and K40 processors are determined as 5.2, 6.4 and 7.4 Flops/Byte respectively.

The numbers given in brackets after each kernel name in Figure 4 give the OI for that kernel. Since all of these are significantly lower than the ridge point values, the Roofline model tells us that the limiting factor is memory bandwidth (rather than floating point capability) without exception. This means that a reasonable method to assess performance portability is to simply to compare the memory bandwidth obtained by each kernel to that obtained by STREAM, on each architecture. Note that STREAM does not report peak bandwidth, but gives an indication of what is practically achievable in a well-performing case, so it is possible for kernels to slightly outperform STREAM.

Figure 4 shows the memory bandwidth of each kernel expressed as a percentage of STREAM. For real, complex applications, it is over-ambitious to expect $100 \%$ across all kernels. The performance will depend on the specific complexities of each kernel, but it is clear that, for a well-performing code, most kernels should be achieving a reasonable percentage of STREAM bandwidth. First considering the K40 GPU results in the Figure, it can be seen that all kernels are near $100 \%$, except for "Chemical Stress". Further investigation reveals that this kernel achieves relatively low occupancy on the GPU because of the register usage required by each thread in efficiently storing temporary structures. Future work will attempt to redesign the kernel at the algorithmic level with the aim of improving performance across all architectures.

We derive estimates of corresponding bandwidths on the Ivy-bridge and Xeon Phi using the measured K40 bandwidth results scaled by ratios of timing results (due to the lack of appropriate tools, on the platforms in use, that can provide the necessary kernel-level information). These are only estimates because they assume that the same amount of data is being loaded from main memory across the three architectures, which may be an oversimplification due to differing caching architectures and policies, but nevertheless give us useful indications. The CPU and Xeon Phi results are seen to lack the consistency of the GPU results, more so for Ludwig (for which the kernels are typically more complex) than MILC. Our estimation technique may play a role in this variability, but is is also possible that the GPU architecture, designed for data throughput, is more effective in achieving good bandwidth for such real applications. Nevertheless, for the CPU, we see $60 \%$ or higher of STREAM bandwidth for all but two kernels. The variability for the Xeon Phi is higher, but this is not surprising since the current product is known 


\begin{tabular}{|c|c|c|}
\hline & Titan & ARCHER \\
\hline \hline Location & $\begin{array}{c}\text { Oak Ridge } \\
\text { National Laboratory }\end{array}$ & $\begin{array}{c}\text { University of } \\
\text { Edinburgh }\end{array}$ \\
\hline Product & Cray XK7 & Cray XC30 \\
\hline Per Node & $\begin{array}{c}\text { One Interlagos \& } \\
\text { one K20X }\end{array}$ & Two Ivy-Bridge \\
\hline Nodes & 18,688 & 4,920 \\
\hline Interconnect & Cray Gemini & Cray Aries \\
\hline
\end{tabular}

Table 2. Technical details of the supercomputers used in the scaling analysis. See Table 1 for details of the processors.

to have architectural limitations which are being addressed by a complete re-design for the next-generation "Knights Landing" product. Since we are already carefully tuning the data layout and corresponding access patterns, it is not clear that there are any major improvements which could be made to the application kernels, but it is possible that tuning of more subtle system settings may be advantageous and we will explore this in future work. Intel quote $80 \mathrm{~GB} / \mathrm{s}$ (around $50 \%$ of STREAM) as a threshold on the current Xeon Phi, above which memory bandwidth can be considered as being good (Cepeda, Shannon 2012), and we are achieving this for 9 of our 13 kernels.

\section{Scaling}

In this paper we have presented our targetDP model, and demonstrated its effectiveness in allowing the same application source code to perform well across modern architectures. The model is relevant for intra-node parallelism, so for use on multi-node systems it must be combined with a higher-level paradigm. Both the applications we have presented use MPI for inter-node communications. In this section, we analyse the performance of our MPI+targetDP applications at scale.

For this analysis we use the Titan and ARCHER supercomputers, for which details are given in Table 2. On each node, Titan has one 16-core Interlagos CPU and one K20X GPU, whereas ARCHER has two 12-core Ivy-bridge CPUs. In this section, we evaluate on a node-by-node basis. For Titan, a single MPI task per node, operating on the CPU, is used to drive the GPU on that node. We also include, for Titan, results just using the CPU on each node without any involvement from the GPU, for comparison. This means that, on a single node, our Titan results will be the same as those $\mathrm{K} 20 \mathrm{X}$ and Interlagos results presented in the previous section (for the same test case). On ARCHER, however, we fully utilize both the processors per node: to do this we use two MPI tasks per node, each with 12 OpenMP threads (via targetDP). So the single node results for ARCHER are twice as fast as those Ivy-bridge single-processor results presented in the previous section. Note that targetDP has no capability to decompose data parallel operations across both CPUs and GPUs within a single run. The advantage of this would be minimal, compared to sole use of the GPU, even if good load balancing was achieved, since the memory bandwidth on the GPU is so much higher than the CPU, so we do not believe it is worth the substantial complexity that would be required. Similar to the previous section, we run for 1000 timesteps or iterations and, since these runs are across multiple nodes on a shared system, we reduce the effects of any interference from other jobs by performing each run several times and select the minimum timing result.

In Figure 5, we show strong scaling, for two different problem sizes, for both Ludwig and MILC. The small problem sizes on the left are those which were used in our single-processor analysis in the previous section. The problem sizes on the right are chosen to be representative of current problems requiring HPC. For each, we see how the time to solution deceases as we increase the number of nodes.

For the small problem cases, on the CPUs, the scaling is seen to be excellent up to around 32 nodes. After that point, the local problem size becomes too small and communication dominates. For the GPU case, strong scaling is still observed up to this point (i.e. the time to solution continues to decrease), but the scaling is seen to deviate from the ideal case. At low node counts the single GPU per node on Titan outperforms the two CPUs per node on ARCHER, but there is a crossover point (around 32 nodes) after which the ARCHER performance is better.

This behaviour is expected to be attributable to the extra data transfers across the PCI-express bus necessary when exchanging halo data between GPUs (via the host CPUs). We expect that hardware improvements in future systems will reduce these overheads: most notably the introduction of the higher bandwidth and lower latency NVLINK as a high performing replacement for PCI-express in future NVIDIA models (NVIDIA Whitepaper 2014). There are also potential improvements in software through use of CUDA-aware MPI, which should reduce overheads through removal of unnecessary data buffering and CPU involvement. This can be used in a straightforward manner by passing pointers to the target rather than host copies of the data structures directly into MPI calls. There already exists such a library on Titan, but it still lacks maturity and is not used here because of incompatibilities with the specific communication patterns used in these applications.

For the larger problem sizes, there exists a minimum number of nodes for which the problem can fit into total memory: 256 nodes for Ludwig and 64 nodes for MILC (i.e. the Ludwig problem size is larger). It can be seen that the behaviour is similar, with the crossover point dependent on the problem size, occurring at around 512 nodes for Ludwig and 256 nodes for MILC.

Overall, these results confirm that targetDP can be combined effectively with MPI for use on large-scale supercomputers for these types of problem.

\section{Conclusions}

Large-scale HPC systems are increasingly reliant on highly parallel processors such as NVIDIA GPUs and Intel Xeon Phi many-core CPUs to deliver performance. It is unsustainable for the programmer to write separate code for each of these different architectures (and also traditional CPUs). In this paper we presented a pragmatic solution to this problem, for grid-based applications. The targetDP model consists of a very lightweight framework, which abstracts either OpenMP (for multi-core or many-core CPUs) or CUDA (for NVIDIA GPUs), whilst allowing good vectorization on the former. We showed that use 

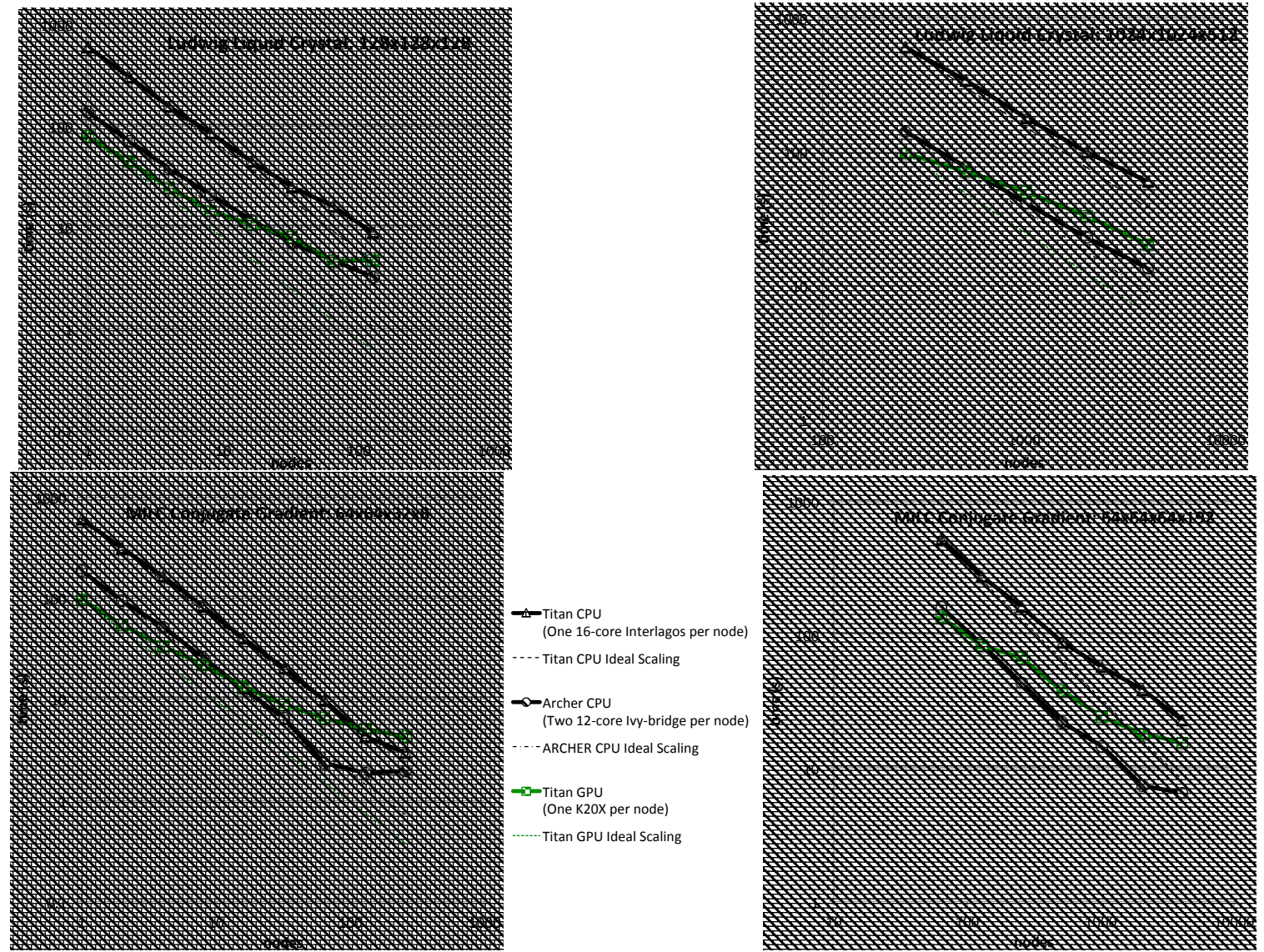

Figure 5. The strong scaling, on Titan and ARCHER, of Ludwig (top) and MILC (bottom) for small (left) and large (right) problem sizes. For ARCHER, both CPUs are used per node. For Titan, we include results with and without GPU utilization.

of this approach can achieve real performance portability across the architectures for complex applications, and that it can effectively be combined with MPI for use on large supercomputers. An important finding is that the explicit vectorization capabilities of the model are vital in order to make efficient use of the current Xeon Phi processors which feature 512-bit SIMD units, and are expected also be vital on imminent mainstream CPU products, for which the width of the vector instruction will increase to this size.

Our work, for which the software is open source and freely available (Gray and Stratford 2016), has so far been limited to structured grid applications, but the model or ideas may well be of interest more widely. The main architecture that we have not yet addressed is the AMD GPU, which we should be able to target in a straightforward manner through development of an OpenCL implementation of targetDP (that largely mirrors the CUDA implementation). It should be similarly straightforward to create Fortran implementations (for the key architectures discussed in this paper) to support the many HPC grid-based applications written in that language. We also look forward to imminent hardware advances from Intel and NVIDIA: the next generation Xeon Phi and GPU products will both exploit high-bandwidth stacked memory, which should significantly boost performance of our memory-bandwidth bound applications. Furthermore, the new low latency and high bandwidth NVLINK CPU to GPU interconnect promises to reduce the communication overheads in strong scaling on GPU systems.

\section{Acknowledgments}

AG acknowledges support from the embedded CSE programme of the ARCHER UK National Supercomputing Service (http://www.archer.ac.uk) and the European Union funded PRACE programme. KS acknowledges support from United Kingdom EPSRC grant EP/J007404. This research used resources of the Oak Ridge Leadership Computing Facility at the Oak Ridge National Laboratory, which is supported by the Office of Science of the U.S. Department of Energy under Contract No. DE-AC05-00OR22725, and the United Kingdom EPSRC funded ARCHER service. We thank Daniel Holmes and Arno Proeme for providing constructive feedback on this paper.

\section{References}

Bauer, M., Treichler, S., Slaughter, E., and Aiken, A. (2012). Legion: Expressing locality and independence with logical regions. In Proceedings of the International Conference on High Performance Computing, Networking, Storage and Analysis, SC '12, pages 66:1-66:11, Los Alamitos, CA, USA. IEEE Computer Society Press. http://dl.acm.org/citation.cfm?id=2388996.2389086.

Beris, A. N. and Edwards, B. J. (1994). Thermodynamics of flowing systems: with internal microstructure.

Bull, M. (2013). PRACE 2IP D7.4, Unified European Application Benchmark Suite. http://www.prace-ri.eu/ueabs/.

Cepeda, Shannon (2012). Optimization and performance tuning for intel xeon phi coprocessors, part 2 . https://software.intel.com/en-us/articles. 
Davies, C. T., Follana, E., Gray, A., Lepage, G., Mason, Q., Nobes, M., Shigemitsu, J., Trottier, H., Wingate, M., Aubin, C., et al. (2004). High-precision lattice qcd confronts experiment. Physical Review Letters, 92(2):022001.

de Gennes, P. and Prost, J. (1995). The physics of liquid crystals. Number 83. Oxford University Press (Clarendon).

Desplat, J.-C., Pagonabarraga, I., and Bladon, P. (2001). LUDWIG: A parallel Lattice-Boltzmann code for complex fluids. Computer Physics Communications, 134(3):273-290.

Edwards, H. C., Sunderland, D., Porter, V., Amsler, C., and Mish, S. (2012). Manycore performance-portability: Kokkos multidimensional array library. Scientific Programming, 20(2):89-114.

Gray, A., Hart, A., Henrich, O., and Stratford, K. (2015). Scaling soft matter physics to thousands of graphics processing units in parallel. International Journal of High Performance Computing Applications, page 1094342015576848.

Gray, A., Hart, A., Richardson, A., and Stratford, K. (2012). Lattice Boltzmann for Large-Scale GPU Systems, pages 167-174. Advances in Parallel Computing. IOS Press.

Gray, A. and Stratford, K. (2013). Ludwig: multiple GPUs for a complex fluid lattice Boltzmann application. Chapman and Hall/CRC.

Gray, A. and Stratford, K. (2014). targetDP: an Abstraction of Lattice Based Parallelism with Portable Performance. In High Performance Computing and Communications, 2014, pages 312-315. IEEE.

Gray, A. and Stratford, K. (2016). targetDP Web page. http://ludwig.epcc.ed.ac.uk/targetdp.

Harris, M. (2016). HEMI Web Page. github.com/harrism/hemi.

Henrich, O., Stratford, K., Coveney, P. V., Cates, M. E., and Marenduzzo, D. (2013). Rheology of cubic blue phases. Soft Matter, 9(43):10243-10256.

Hornung, R., Keasler, J., et al. (2014). The RAJA portability layer: overview and status. Lawrence Livermore National Laboratory, Livermore, USA.

Khronos OpenCL Working Group (2015). OpenCL Specification. Version 2.1. https://www.khronos.org/opencl.

Khronos OpenCL Working Group - SYCL Subgroup (2015). SYCL Specification. Version 1.2. https://www.khronos.org/sycl.

McCalpin, J. D. (1995). Memory bandwidth and machine balance in current high performance computers. IEEE Computer Society Technical Committee on Computer Architecture (TCCA) Newsletter, pages 19-25.
McIntosh-Smith, S., Boulton, M., Curran, D., and Price, J. (2014). On the performance portability of structured grid codes on many-core computer architectures. In Supercomputing, pages 53-75. Springer.

Medina, D. S., St-Cyr, A., and Warburton, T. (2014). Occa: A unified approach to multi-threading languages. arXiv preprint arXiv: 1403.0968

NVIDIA Whitepaper (2014). NVIDIA NVLink High-Speed Interconnect: Application Performance. http://www.nvidia.com/object/nvlink.html.

OpenMP Architecture Review Board (2015). OpenMP application program interface version 4.5. http://openmp.org/wp/openmpspecifications/.

Slaughter, E., Lee, W., Treichler, S., Bauer, M., and Aiken, A. (2015). Regent: a high-productivity programming language for hpc with logical regions. In Proceedings of the International Conference for High Performance Computing, Networking, Storage and Analysis, page 81. ACM.

Steuwer, M., Fensch, C., Lindley, S., and Dubach, C. (2015). Generating performance portable code using rewrite rules: from high-level functional expressions to high-performance OpenCL code. In Proceedings of the 20th ACM SIGPLAN International Conference on Functional Programming, pages 205-217. ACM.

Stratford, K., Gray, A., and Lintuvuori, J. (2015). Large colloids in cholesteric liquid crystals. Journal of Statistical Physics, 161(6):1496-1507.

Succi, S. (2001). The lattice Boltzmann equation: for fluid dynamics and beyond. Oxford university press.

The MILC Collaboration (2014). The MILC Code manual. http://www.physics.utah.edu/ detar/milc/.

The OpenACC Standard Committee (2015). The OpenACC Application Programming Interface Version 2.5. http://www.openacc.org/.

Tiribocchi, A., Henrich, O., Lintuvuori, J., and Marenduzzo, D. (2014). Switching hydrodynamics in liquid crystal devices: a simulation perspective. Soft matter, 10(26):4580-4592.

Volkov, V. (2010). Better performance at lower occupancy. In Proceedings of the GPU Technology Conference, GTC, volume 10. San Jose, CA.

Williams, S., Waterman, A., and Patterson, D. (2009). Roofline: an insightful visual performance model for multicore architectures. Communications of the ACM, 52(4):65-76. 